21st Particles and Nuclei International Conference (PANIC 2017)

International Journal of Modern Physics: Conference Series

Vol. 46 (2018) 1860058 (6 pages)

(C) The Author(s)

DOI: $10.1142 / \mathrm{S} 2010194518600583$

\title{
Search for Heavy Higgs Bosons in Fermionic Decay Channels with CMS
}

\author{
Ye Chen* (on behalf of the CMS Collaboration) \\ Institute of High Energy Physics, Chinese Academy of Sciences \\ Beijing, Shijingshan, 100049, China
}

Published 3 May 2018

\begin{abstract}
Latest results of searches for heavy Higgs bosons in fermionic final states are presented using the CMS detector at the LHC. Results are based on pp collision data collected at centre-of-mass energies of 8 and $13 \mathrm{TeV}$ which have been interpreted according to different extensions of the Standard Model such as MSSM, 2HDM, and NMSSM. These searches look for evidence of other scalar or pseudoscalar bosons, in addition to the observed SM-like $125 \mathrm{GeV}$ Higgs boson, and set $95 \%$ confidence level upper limits in fermionic final states and benchmark models explored. The talk reviews briefly the major results obtained by the CMS Collaboration during Run I, and presents the most recent searches performed during Run II.
\end{abstract}

Keywords: CMS; Heavy Higgs Bosons; Fermionic Decay; MSSM; 2HDM; NMSSM.

\section{Introduction: Heavy Higgs Boson Searches at CMS}

The Minimal Supersymmetric Standard Model (MSSM) was originally proposed around the eighties of the last century ${ }^{1,2,3}$. Within the MSSM theory, two Higgs doublet states are allowed, resulting in five Higgs boson final states which include two spin even neutral Higgs bosons $(\mathrm{h} / \mathrm{H})$, one spin odd neutral Higgs boson $(\mathrm{A})$ and two charged Higgs bosons $\left(H^{ \pm}\right)$. After the discovery of the standard model like Higgs boson, the MSSM model has become one of the benchmark scenarios for additional Higgs boson searches at the $\mathrm{CMS}^{4}$ experiment. A recent summary of MSSM Higgs boson production cross sections can be found in the LHC Higgs boson Cross Section Working Group (HXSWG) report ${ }^{5}$. The progress of MSSM Higgs boson searches also triggers prospective Higgs boson like resonance searches in other phenomenological models, including the Two Higgs Doublet model $(2 \mathrm{HDM})^{6}$, Graviton models ${ }^{7}$ etc.

aThe speaker is supported by (a) Young Scholar Grant from the National Natural Science Foundation of China as Principal Investigator under Contract No.11405194; (b) DESY ONACPR exchange postdoctoral fellowship, from November 2014 to November 2016; (c) National Natural Science Foundation of China under Contract No. 11461141011; (d) Ministry of Science and Technology of China, 973 project under Contract No. 2013B837801.

This is an Open Access article published by World Scientific Publishing Company. It is distributed under the terms of the Creative Commons Attribution 4.0 (CC-BY) License. Further distribution of this work is permitted, provided the original work is properly cited. 

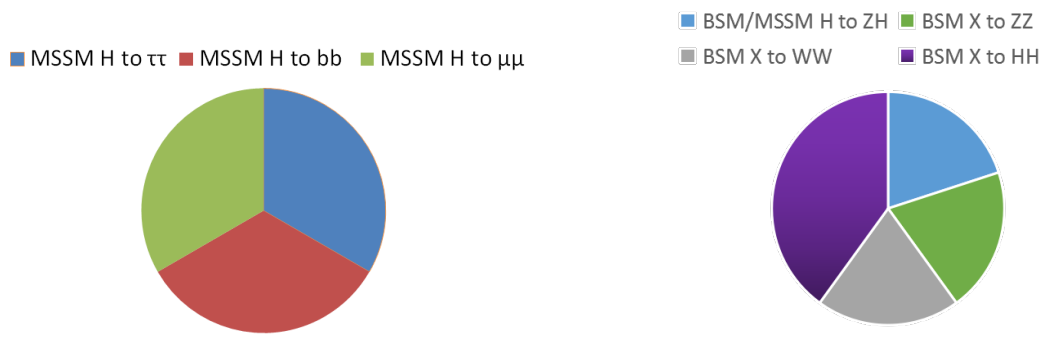

Fig. 1. Neutral heavy higgs decay modes being explored with the CMS experiment.

Since 2011, the CMS Collaboration has written publications specifically targeting MSSM Higgs boson searches taken at three center-of-mass energies. As illustrated in Fig. 1, neutral Higgs bosons have been searched via three fermionic final states $\tau \tau^{8,9,10,11}, b b^{13,14}$, and $\mu \mu^{15}$. There are additional neutral Higgs boson or resonance searches via double bosonic decays like $Z H, H H, Z Z$ and $W W$, with results partially re-interpreted within the framework of MSSM scenarios ${ }^{18}$. The charged Higgs boson searches have been performed via final states of $\tau \nu$ and $t b^{16}$, which could be very important for further constraints of the MSSM parameter space.

\section{Neutral MSSM Higgs Boson search via $\tau \tau$}

For the neutral MSSM Higgs boson production, the cross section is quite low. However, when a Higgs boson is produced associated with a b quark, the cross section could be enhanced proportional to $\tan ^{2} \beta$. There are three di-fermion decay modes that have been discussed in this session, namely $\tau \tau, b \bar{b}$, and $\mu \mu$. In Fig. 2, three principal tree level production subprocesses are depicted.

The search for neutral MSSM Higgs bosons via $\tau \tau$ decays has been performed by the CMS experiment ${ }^{8,9}$ with $7 \mathrm{TeV}$ and $8 \mathrm{TeV}$ data. The decay mode has been re-investigated with around $2.3 \mathrm{fb}^{-1}$ data collected in $2015^{10}$ and then with 12.9 $\mathrm{fb}^{-1}$ data collected in $2016^{11}$. Four decay modes of $\tau \tau$ pairs have been studied, $\mu \tau_{h}$, $e \tau_{h}, e \mu$ and $\tau_{h} \tau_{h}$. The final transverse invariant mass distribution of $\mu \tau_{h}$ has been shown in Fig. 3 as an example. The combination results have reached the region beyond $1 \mathrm{TeV}$ and there are no obvious new phenomena that have been seen in the
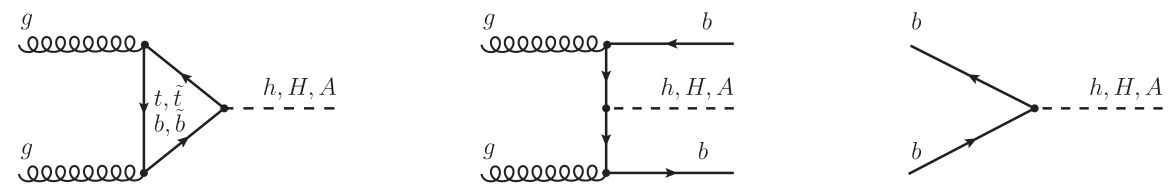

Fig. 2. Feynman diagrams of neutral Higgs boson production in the MSSM framework. Left: leading order MSSM Higgs boson production with the gluon fusion process. Middle: leading order MSSM b-associated Higgs boson production. Right: additional leading order MSSM Higgs boson production with the five-flavor scheme $8,9,10,11$. 


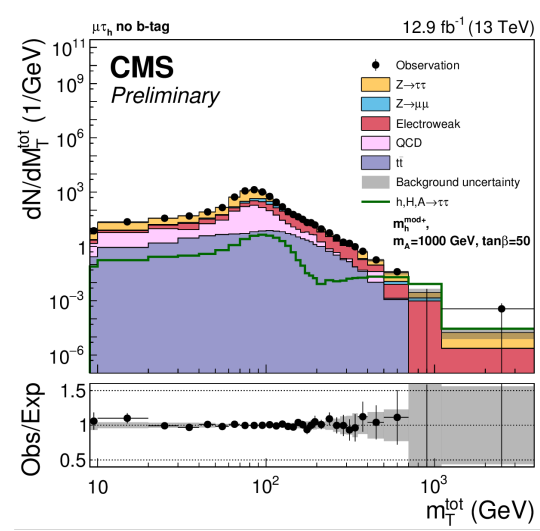

(a)

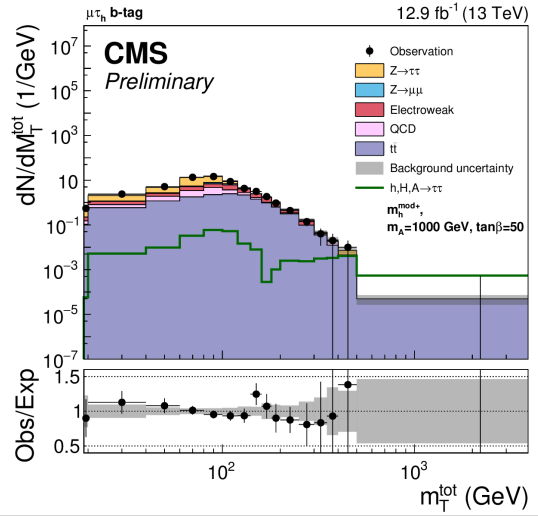

(b)

Fig. 3. MSSM $H \rightarrow \tau \tau$ search with $12.9 \mathrm{fb}^{-1} 13 \mathrm{TeV}$ data. Post-fit plots of the $\tau \tau$ transverse mass distribution in the $\mu \tau_{h}$ channel. The left plot is without the b-tag category and the right plot is with the b-tag category ${ }^{11}$.

$\tau \tau$ spectrum. A model independent limit has been provided, along with the MSSM $\bmod _{h}^{\text {mod+ }}$ and hMSSM scenarios in a CMS Physics Analysis Summary ${ }^{11}$. With all data collected in 2016, the parameter spaces of $M_{A}$ and $\tan \beta$ have been further constrained $^{12}$.

\section{Neutral MSSM Higgs Boson search via $b \bar{b}$}

The $b \bar{b}$ final state has been considered as a very important decay mode for Higgs boson searches due to the relative large branching ratio of the Higgs boson decay to $b \bar{b}$. The large QCD multi-jet background made this channel very challenging to measure. For $7 \mathrm{TeV}$ and $8 \mathrm{TeV}$ center-of-mass energies, two results have been released by the CMS Collaboration ${ }^{13,14}$. $95 \%$ confidence level upper limits of MSSM Higgs bosons produced in association of at least one $\mathrm{b}$ quark and decaying to $b \bar{b}$ have been set. The physics results have been discussed for several MSSM benchmark scenarios and an upper limit of the Higgs boson cross section has been provided combining the $7 \mathrm{TeV}$ and $8 \mathrm{TeV}$ CMS data. Until now, the CMS results are the only results for the search of the MSSM Higgs boson to $b \bar{b}$ in LHC experiments.

\section{Neutral MSSM Higgs Boson search via $\mu \mu$}

The MSSM Higgs boson to $\mu \mu$ decay mode has been presented with relatively low systematic uncertainties ${ }^{15}$. Fig. 5 shows the invariant spectrum of $\mu \mu$ without or with b-tag. The combined 7 and $8 \mathrm{TeV}$ results have been presented together with the $m_{h}^{\bmod +}$ MSSM scenario in Ref. ${ }^{15}$. Due to the fact that the cross section is around $10^{-3}$ lower compared to $\tau \tau$ decays, the discovery region beyond $1 \mathrm{TeV}$ has not yet been accessible with currently available results. With data accumulated in Run II, 

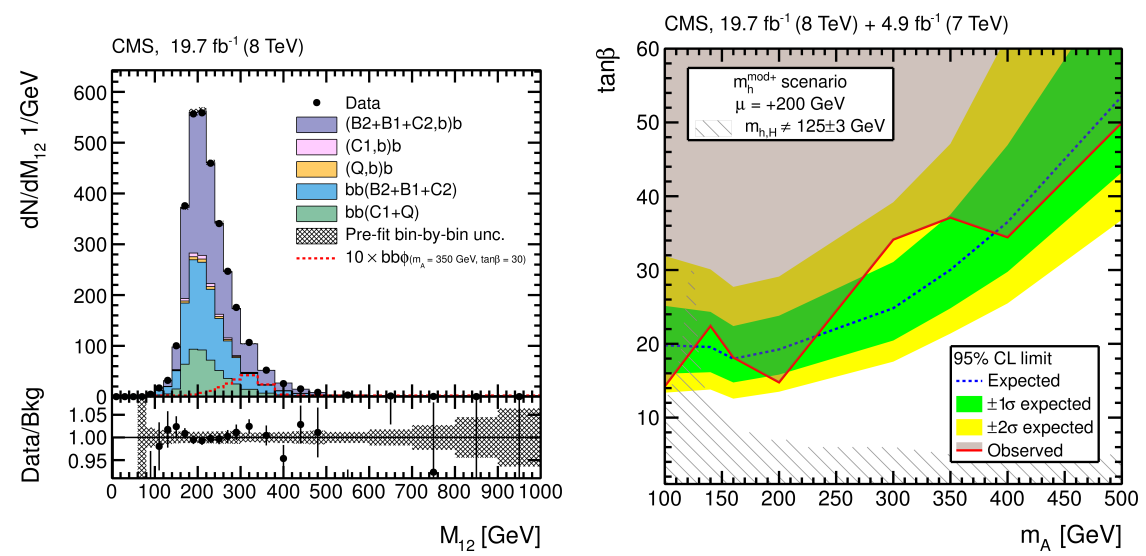

Fig. 4. MSSM $H \rightarrow b \bar{b}$ search with $19.6 \mathrm{fb}^{-1} 8 \mathrm{TeV}$ data. Left: the invariant mass spectrum of the two leading b jets. Right: upper limits of the MSSM Higgs boson cross section in $m_{A}$ and $\tan \beta$ parameter spaces ${ }^{14}$.
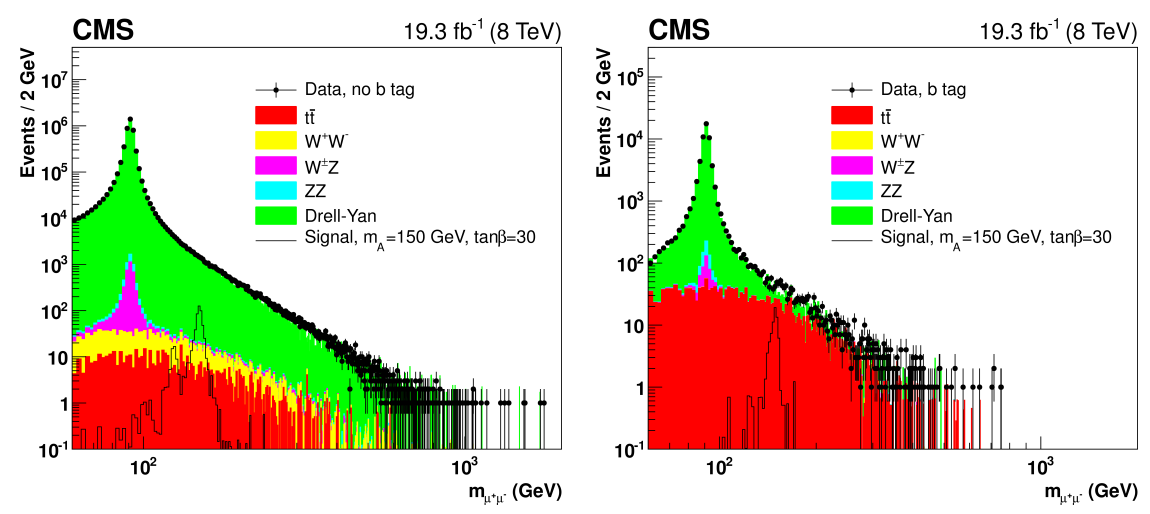

Fig. 5. MSSM $H \rightarrow \mu \mu$ search with $19.6 \mathrm{fb}^{-1} 8 \mathrm{TeV}$ data. Invariant mass spectrum of $\mu \mu$ without or with additional b-tag ${ }^{15}$.

this decay mode could be very attractive given the excellent muon reconstruction performance of the CMS experiment.

\section{Charged MSSM Higgs Boson search}

The MSSM model allows for the existance of two charged Higgs bosons. When $m_{H^{ \pm}}<\left(m_{t}-m_{b}\right)$, a large fraction of charged Higgs bosons are expected to decay via $\tau \nu$; when $m_{H^{ \pm}}$is above the $\left(m_{t}-m_{b}\right)$ threshold, $H^{ \pm} \rightarrow t b$ is expected to provide the dominated production. CMS has performed the charged Higgs boson search through two decay modes $H^{ \pm} \rightarrow \tau \nu$ and $H^{ \pm} \rightarrow t b^{16,17}$. With $19.7 \mathrm{fb}^{-1} 8 \mathrm{TeV}$ Run I data, the upper limits of the cross section for $m_{H^{ \pm}}=80-160 \mathrm{GeV}$ have been set to be $\sigma\left(p p \rightarrow t(b) H^{ \pm}\right) B\left(H^{ \pm} \rightarrow \tau \nu_{\tau}\right)=0.38$ to $0.025 \mathrm{pb}$. Assuming $\mathrm{B}\left(H^{ \pm} \rightarrow t b\right)$ $=1$, upper limits on the cross section $\left(\sigma\left(p p \rightarrow t(b) H^{ \pm}\right)\right.$for $m\left(H^{ \pm}\right)=180-600$ 


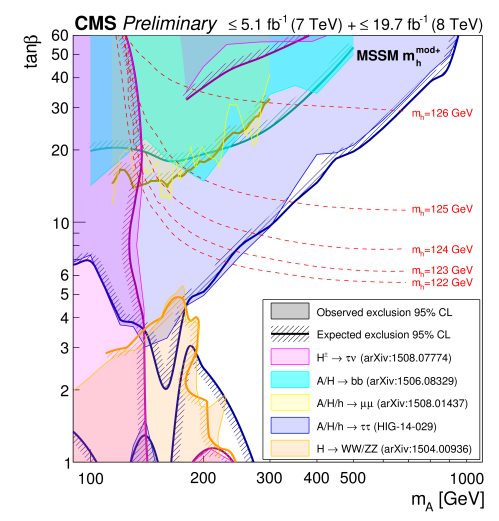

Fig. 6. Combined $m_{h}^{\text {mod }+}$ MSSM results with CMS 7 and $8 \mathrm{TeV}$ data ${ }^{18}$.

GeV are set to be 2.0 to $0.13 \mathrm{pb}^{16}$. With $12.9 \mathrm{fb}^{-1}$ data accumulated at Run II, the upper limits on charged Higgs bosons times branching ratio are set for Higgs bosons with $180 \mathrm{GeV}<m\left(H^{ \pm}\right)<3 \mathrm{TeV}^{17}$.

\section{Summary and outlook}

An overview of the CMS Run I additional heavy Higgs boson searches has been given in a CMS Physics Ananlysis Summary ${ }^{18}$, including the discussion within MSSM benchmark scenarios. Fig. 6 shows results for one of the MSSM scenarios $m_{h}^{m o d+}$ as an example. Di-fermion processes are currently sensible to high $\tan \beta$ regions and di-boson processes are sensible to low $\tan \beta$ regions.

Searches for heavy Higgs bosons motivated by the predictions of a few models beyond the SM, have become a focus of the CMS experiment in Run II of the LHC after the discovery of the SM-like Higgs boson. New results of fermionic decay modes with around $12.9 \mathrm{fb}^{-1}$ data accumulated in 2016 have been shown along with the Run I reviews. With the current data sample, no hint for new physics has appeared yet. More decay modes both for additional neutral Higgs bosons and charged Higgs bosons are expected to be explored with 2016 and 2017 data.

\section{Acknowledgments}

Many thanks to PANIC 2017 LOC for holding this wonderful conference. The speaker is also grateful to the CMS conference committee for granting the talk opportunity.

Many thanks to the CMS Higgs conveners Paolo Meridiani and Roberto Salerno for helpful revisions. Many thanks to Claudia Wulz, the CMS editor of this proceeding, for the final polishing. The speaker would also like to thank colleagues from the CMS Higgs group (from the Hbb subgroup, especially), the CMS Trigger Study 
group and the IHEP CMS group for inspiration and helpful discussions. Special thanks to CERN for hosting and support during the speaker's residence at CERN.

\section{References}

1. S. Dimopoulos, H. Georgi, 'Softly broken supersymmetry and SU(5)', Nucl. Phys. B 193, $150(1981)$

2. S. Martin, 'A Supersymmetry Primer', arxiv: hep-ph/9709356 (1997)

3. D. Chung, L. Everett, C. Kane, S. King, J. Lykken, L. Wang, 'The soft supersymmetrybreaking Lagrangian: theory and applications', Phys. Rept. 407, 1 (2005)

4. CMS Collaboration, 'The CMS Experiment at the CERN LHC', JINST 3, S08004 (2008)

5. E. Bagnaschi, et. al., 'Benchmark scenarios for low $\tan \beta$ in the MSSM', LHCHXSWG2015-002, (2015)

6. G. C. Branco, et. al., 'Theory and phenomenology of two-Higgs-doublet models', Phys. Rep. 516. 1, (2012)

7. L. Randall, R. Sundrum, 'An Alternative to Compactification', Phys. Rev. Lett. 83, 4690-4693, (1999)

8. CMS Collaboration, 'Search for Neutral Minimal Supersymmetric Standard Model Higgs Bosons Decaying to Tau Pairs in pp Collisions at $\sqrt{s}=7 \mathrm{TeV}$ ', Phys. Rev. Lett. 106, 231801, (2011)

9. CMS Collaboration, 'Search for neutral Higgs boson decaying to a pair of tau leptons in pp collisions', JHEP, 10, 160, (2014)

10. CMS Collaboration, 'MSSM Higgs to tautau search at $13 \mathrm{TeV}$ using 2015 data sample', CMS-PAS-HIG-16-006, (2016), http://cds.cern.ch/record/2160252

11. CMS Collaboration, 'Search for a neutral MSSM Higgs to $\tau \tau$ with $12.9 \mathrm{fb}^{-1}$ of data at $\sqrt{s}=13 \mathrm{TeV}$ ', CMS-PAS-HIG-16-037, (2016), http://cds.cern.ch/record/2231507

12. CMS Collaboration, 'Search for additional neutral MSSM Higgs bosons in the ditau final state in pp collisions at $\sqrt{s}=13$ TeV', CMS-PAS-HIG-17-020, (2017), http://cds.cern.ch/record/2296333

13. CMS Collaboration, 'Search for a Higgs boson decaying into a b-quark pair and produced in association with b quarks in proton-proton collisions at $7 \mathrm{TeV}$ ', Phys. Lett. B 722, 207 (2013).

14. CMS Collaboration, 'Search for neutral MSSM Higgs bosons decaying into a pair of bottom quarks', JHEP 1511, 071 (2015)

15. CMS Collaboration, 'Search for neutral MSSM Higgs bosons decaying into $\mu^{+} \mu^{-}$in pp collisions at $\sqrt{S}=7 \mathrm{TeV}$ and $8 \mathrm{TeV}$ ', Phys. Lett. B 752, 221 (2016).

16. CMS Collaboration, 'Search for a charged Higgs boson in pp collisions at $\sqrt{s}=8$ TeV', JHEP 1511, 018 (2015)

17. CMS Collaboration, 'Search for charged Higgs bosons with the $H^{ \pm} \rightarrow \tau^{ \pm} \nu_{\tau}$ decay channel in the fully hadronic final state at $\sqrt{s}=13$ TeV', CMS-PAS-HIG-16-031 (2016), http://cds.cern.ch/record/2223865

18. CMS Collaboration, 'Summary results of high mass BSM Higgs searches using CMS run-I data', CMS-PAS-HIG-16-007 (2016), http://cds.cern.ch/record/2142432 